\title{
The effective layer height for star wandering and the accuracy of tilt sensing in multicolour laser stars
}

\author{
R. Ragazzoni ${ }^{1,2}$, E. Marchetti ${ }^{2}$, and G. Brusa ${ }^{3}$ \\ 1 Astronomical Observatory of Padova, vicolo dell'Osservatorio 5, I-35122 Padova, Italy \\ e-mail: ragazzoni@astrpd.pd.astro.it \\ 2 CISAS, vicolo dell'Osservatorio 5, I-35122 Padova, Italy \\ e-mail: marchetti@astrpd.pd.astro.it \\ 3 Center for Astronomical Adaptive Optics, 933 N Cherry Ave. Tucson, U.S.A. \\ e-mail: gbrusa@as.arizona.edu
}

Received April 1; accepted June 20, 1996

\begin{abstract}
Due to the finite height of the mesospheric sodium laser guide star, it is shown that the measurement of absolute tilt done with the multi-colour laser star technique is affected by an uncertainty depending upon the $C_{n}^{2}$ distribution. Two case studies for $D=3.58$ and $D=8.00$ meter telescopes are given.
\end{abstract}

Key words: atmospheric effects - techniques: miscellaneous - telescopes

\section{Introduction}

In the next years a number of telescopes with aperture diameter much larger than the existing HST will see their first-light (Mountain et al. 1994). In this framework the Adaptive Optics (AO) technique (Beckers 1993) can provide a substantial improvement in the resolution of optical imaging in the next decades, provided that a reliable way to obtain routinely $\mathrm{AO}$ compensated images in the visible becomes available. While it has been already pointed out that using nearby Natural Guide Stars (NGSs) as reference does not allow for a full sky coverage, several techniques have been proposed in order to overcome the problem. Mesospheric Sodium Laser Guide Stars (LGSs), Foy \& Labeyrie (1985), has been proven to be effective to close an AO control loop (Olivier et al. 1995). The first scientific results with Rayleigh LGSs have been published in the astronomical literature (Drummond et al. 1995; McCullough et al. 1995). The problems of conical anisoplanatism are well studied (Fried \& Belsher 1994) and the solutions to the problems arose have been selected (Tallon \& Foy 1990). LGS technology is, in practice, still in its infancy, and it is realistic to expect that in a decade these

Send offprint requests to: R. Ragazzoni systems will be routinely available for most of the modern telescopes. The tilt indetermination problem (Pilkington 1987; Olivier et al. 1993), however, still requires a convincing solution: the Double Adaptive Optics (Rigaut \& Gendron 1992) increases significantly the sky coverage, but the full sky is not obtained; the tristatic configuration (Belen'kii 1995; Ragazzoni 1996a) requires huge modifications on the telescope structure; the auxiliary telescopes technique (Ragazzoni et al. 1995) needs a large available space in the neighborouds of the observatory (Marchetti \& Ragazzoni 1996), the propagation delay technique imposes to share the telescope aperture with the laser projector (Ragazzoni 1996b) and the multicolour LGS (Foy et al. 1992, 1995) requires a large laser power.

While one can hope that in the near future other techniques, or a combination of existing ones, should lead to a reliable, routinely usable, full AO compensation of any point in the sky, a careful analysis of the existing techniques should be carried out. In the following a detailed analysis of an uncovered aspect of the multicolour LGS technique is carried out.

\section{The single layer case}

In this section it is assumed that only a single, thin layer, is responsible for the star wandering. We recall here that the multicolour LGS absolute tilt determination technique is based on the measurement of the differential angle of arrival of two different colour LGSs excited by a monochromatic laser beam. Following Foy et al. (1995), when the layer is introducing a tilt $\alpha$ at a given primary wavelenght, the two colour beams will experience a deflection's difference $\gamma$ given by:

$\gamma=\frac{\Delta n}{n-1} \alpha$ 
where $n$ is the refractive index at the primary wavelength and $\Delta n$ is the variation of refractive index of the air between the two LGS colours. The tilting layer is placed at height $h$ while the LGS is excited at height $H$. While the tilting layer can take place in any $h=0 . .20 \mathrm{~km}$, the mesospheric Sodium LGS exhibits a characteristic height $H=95 \mathrm{~km}$ (Happer et al. 1994). In the following we refer to RED and BLUE respectively to the longest and shortest wavelengths of the two LGS colours. Moreover, we'll assume that the exciting beam is a red one, and that the LGS will produce both a red and a blue beam; for sake of simplicity we also assume that the laser firing and the $\mathrm{AO}$ observations are performed at the local zenith. None of these assumptions will affect significantly the conclusions.

Referring to Fig. 1, we consider the chief ray of the beam from the LGS to the observing telescope, where the pupil is located. The $x_{*}$ position of the LGS following the

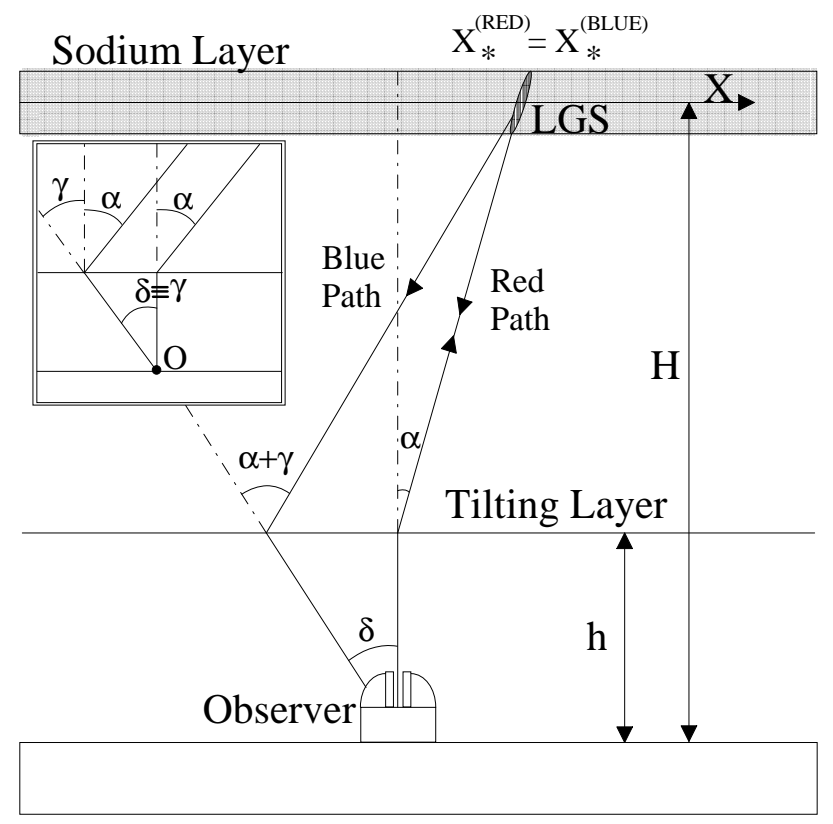

Fig. 1. The apparent displacement $\delta$ between two colour artificial beacons as seen by the ground observer is slightly different than the quantity $\gamma$. Their ratio depends upon the $h$ altitude. It is also shown in the upper-left corner inset how, assumming $H \rightarrow \infty$, the measured angle $\delta$ strictly coincides with $\gamma$

two colour beams can be easily derived under the assumption that all the considered angles are very small.

For the red beam:

$x_{*}^{(\mathrm{RED})} \approx \alpha(H-h)$

while, following the blue laser beam path:

$x_{*}^{(\mathrm{BLUE})} \approx-\delta \cdot h+(\alpha+\gamma-\delta)(H-h)$.
Equating the expressions given in Eqs. (2) and (3) we get:

$\delta=\gamma\left(\frac{H-h}{H}\right)=\alpha \frac{\Delta n}{n-1}\left(\frac{H-h}{H}\right)$.

From Eq. (4) one can see that the measurement $\delta$ will depend upon both $\alpha$ and $h$. In the case of a single layer with a constant altitude this will translate into the introduction of a scale factor close to 1 . In fact, $h$ is usually less than $\frac{1}{5} H$. However, a time evolving value for $h$ will introduce an apparent relative displacement $\delta$ of the red and blue beacons undistinguishable from the displacement introduced by the temporal evolution of the tilt $\alpha$. One can expect that, using the more realistic multi-layer approach, some spreading of the effective $h$ can introduce an error source in the absolute tilt determination.

\section{The multi-layer case}

In this case (see Fig. 2) every layer introduces a tilt term $\alpha_{i}$. For each of these layers one can define a $\gamma_{i}$ using Eq. (1). The position of the LGS can be obtained, in the

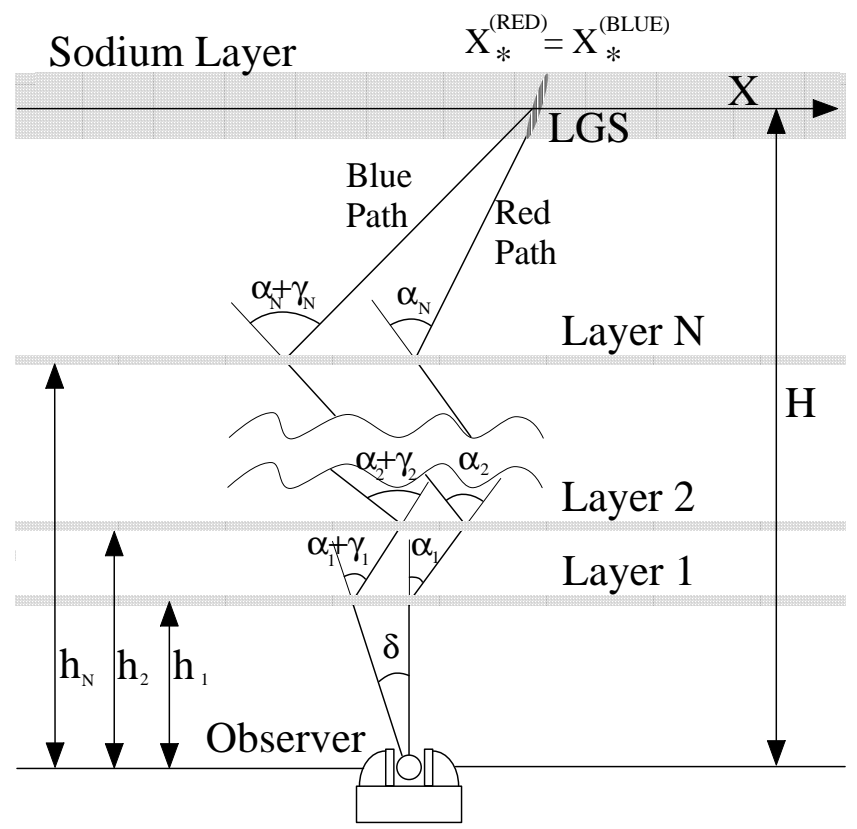

Fig. 2. Also in the multi-layer case a tiny difference between $\delta$ and $\sum \gamma_{i}$ will be experienced

previous approximation, by:

$x_{*}^{(\mathrm{RED})}=\sum_{i} \alpha_{i}\left(H-h_{i}\right)$

for the red beam path, while following the blue beam:

$x_{*}^{(\mathrm{BLUE})}=-\delta \cdot H+\sum_{i}\left(\alpha_{i}+\gamma_{i}\right)\left(H-h_{i}\right)$. 
As usual, equating the expressions given by Eqs. (5) and (6) the apparent displacement $\delta$ is obtained as:

$\delta=\sum_{i} \gamma_{i}\left(\frac{H-h_{i}}{H}\right)$.

Using Eq. (1) the latter can be written as:

$\delta=\frac{\Delta n}{n-1} \sum_{i} \alpha_{i}\left(\frac{H-h_{i}}{H}\right)$.

Using Eq. (8) we define an effective tilting height $\hat{h}$ such that:

$\delta=\alpha \frac{\Delta n}{n-1}\left(\frac{H-\hat{h}}{H}\right)$

where $\alpha=\sum \alpha_{i}$ is the overall tilt at the primary wavelength. Equating the expressions given in Eqs. (8) and (9) one obtains:

$\hat{h}=\frac{\sum_{i} \alpha_{i} h_{i}}{\sum_{i} \alpha_{i}}$.

It is easy to realize that such effective tilting height $\hat{h}$ is a time-dependent parameter. Moreover, it is to be pointed out that $\hat{h}$ is allowed to have any value between $-\infty$ and $+\infty$ : when, occasionally, $\alpha \rightarrow 0$ the result of Eq. (10) will diverge.

In the established conditions there is no way to retrieve the current value for $\hat{h}$. However one can think to introduce a mean effective height $\langle\hat{h}>$ and use such a number to deduce the overall tilt $\alpha$. Using this approach some error $\varepsilon$ will be introduced and one can write:

$\alpha+\varepsilon=\delta \frac{n-1}{\Delta n}\left(\frac{H-<\hat{h}>}{H}\right)$

and using Eq. (9) the error $\varepsilon$ can be worked out:

$\varepsilon=\alpha \frac{<\hat{h}>-\hat{h}}{H-<\hat{h}>}$.

One can see that in the limiting case $\hat{h} \rightarrow<\hat{h}>$ (a single layer) or $H \rightarrow \infty$ (the case of an NGS), the error $\varepsilon$ will become zero.

\section{Applications to atmosphere models}

We have performed a number of simulations using the 4layers fit of Roggermann et al. (1995) to various models published in the literature, rescaling them for different values of $r_{0}$. Namely the Greenwood good seeing model (Greenwood 1977) is denoted by G-77; the fit with Maui (Hawaii) U.S. Air Force Optical Station (Miller et al. 1976) is denoted by SLC-N and the Hufnagel-Valley model (Hufnagel 1974) with an upper atmospheric wind of 54 miles per hour $\left(\approx 24 \mathrm{~m} \cdot \mathrm{s}^{-1}\right)$ is denoted by HV -54 .
We have generated series of 65536 tilts for each of the four layers in a grid of 6 different $r_{0}$ values for each of the 3 different atmosphere models adopted. For each layer a normal distributed tilt with a dispersion given by (Sarazin \& Roddier 1990):

$\sigma=0.42 \frac{\lambda}{D^{1 / 6} r_{0}^{5 / 6}}$

has been calculated and propagated up to the mesospheric Sodium layer. The effective tilt height has been computed together with the residual jitter for each of the 65536 timepoints taken into consideration. As it can be easily seen
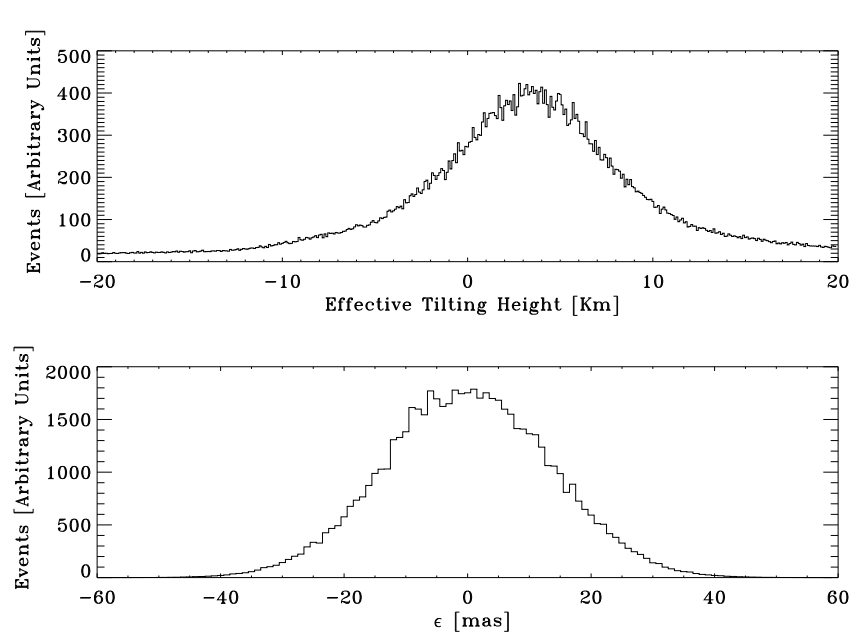

Fig. 3. The histograms for $\hat{h}$ and $\varepsilon$ for $D=8 \mathrm{~m}, r_{0}=0.10 \mathrm{~m}$ in the HV-54 model. The run is composed by 65536 realizations. As it can be seen $\varepsilon$ follows roughly a Gaussian distribution, while the $\hat{h}$ distribution is much more platicurtic

in Fig. 3 the distribution of the effective height is far from a Gaussian shape; however the final residual tilt $\varepsilon$ distribution is very well fitted by a Gaussian distribution, so that it can be identified by the single figure of the dispersion $\sigma_{\varepsilon}$. Following Sandler et al. (1994) one can determine the Strehl ratio degradation contribution due only to the problem pointed out in this paper using the relationship:

$S R \approx\left[1+\frac{\pi^{2}}{2}\left(\frac{\sigma}{\lambda / D}\right)^{2}\right]^{-1}$

The results of the simulation are collected in Tables 1 and 2 and in Fig. 4 for the cases of $D=3.58 \mathrm{~m}$ and $D=8.00 \mathrm{~m}$ telescopes; the fluctuations of the mean effective height $\langle\hat{h}\rangle$ are due to the different realizations of the same multi-layer models. In order to get a deeper insight on the meanings of the published numbers it is here recalled that the diffraction limit capabilities for a $\lambda=500 \mathrm{~nm}$ wavelengths, $\lambda / D$ are respectively 33.9 mas and 15.2 mas for the two telescope aperture taken into consideration. 
Table 1. The results for the perfomed simulation in the $D=3.58 \mathrm{~m}$ case

\begin{tabular}{lrrrrrr}
$r_{0}[\mathrm{~m}]$ & 0.05 & 0.10 & 0.15 & 0.20 & 0.25 & 0.30 \\
\hline $\mathbf{S L C}-\mathbf{N}$ & & & & & & \\
$<\hat{h}>[\mathrm{m}]$ & 1520 & 1530 & 1540 & 1510 & 1520 & 1560 \\
$\sigma_{\varepsilon}[\mathrm{mas}]$ & 13.3 & 7.5 & 5.3 & 4.2 & 3.5 & 3.0 \\
$S R$ & 0.568 & 0.805 & 0.892 & 0.929 & 0.950 & 0.963 \\
\hline $\mathbf{G}-\mathbf{7 7}$ & & & & & & \\
$<\hat{h}>[\mathrm{m}]$ & 798 & 779 & 778 & 797 & 792 & 765 \\
$\sigma_{\varepsilon}[\mathrm{mas}]$ & 10.8 & 6.1 & 4.3 & 3.4 & 2.8 & 2.4 \\
$S R$ & 0.666 & 0.862 & 0.926 & 0.953 & 0.967 & 0.976 \\
\hline $\mathbf{H V}-\mathbf{5 4}$ & & & & & & \\
$<\hat{h}>[\mathrm{m}]$ & 3410 & 3410 & 3380 & 3410 & 3360 & 3390 \\
$\sigma_{\varepsilon}[\mathrm{mas}]$ & 27.2 & 15.3 & 10.9 & 8.6 & 7.1 & 6.1 \\
$S R$ & 0.239 & 0.498 & 0.662 & 0.759 & 0.822 & 0.862
\end{tabular}

Table 2. The same as for Table 1 , in the $D=8.00 \mathrm{~m}$ telescope case

\begin{tabular}{lrrrrrr}
$r_{0}[\mathrm{~m}]$ & 0.05 & 0.10 & 0.15 & 0.20 & 0.25 & 0.30 \\
\hline $\mathbf{S L C}-\mathbf{N}$ & & & & & & \\
$<\hat{h}>[\mathrm{m}]$ & 1540 & 1530 & 1540 & 1520 & 1520 & 1540 \\
$\sigma_{\varepsilon}[\mathrm{mas}]$ & 11.7 & 6.5 & 4.7 & 3.7 & 3.0 & 2.6 \\
$S R$ & 0.255 & 0.526 & 0.679 & 0.774 & 0.839 & 0.874 \\
\hline $\mathbf{G}-\mathbf{7 7}$ & & & & & & \\
$<\hat{h}>[\mathrm{m}]$ & 770 & 791 & 772 & 780 & 791 & 785 \\
$\sigma_{\varepsilon}[\mathrm{mas}]$ & 9.4 & 5.3 & 3.8 & 3.0 & 2.5 & 2.1 \\
$S R$ & 0.346 & 0.625 & 0.764 & 0.839 & 0.882 & 0.914 \\
\hline $\mathbf{H V}-\mathbf{5 4}$ & & & & & & \\
$<\hat{h}>[\mathrm{m}]$ & 3370 & 3400 & 3317 & 3370 & 3313 & 3400 \\
$\sigma_{\varepsilon}[\mathrm{mas}]$ & 23.8 & 13.4 & 9.5 & 7.5 & 6.3 & 5.3 \\
$S R$ & 0.076 & 0.207 & 0.342 & 0.454 & 0.541 & 0.625
\end{tabular}
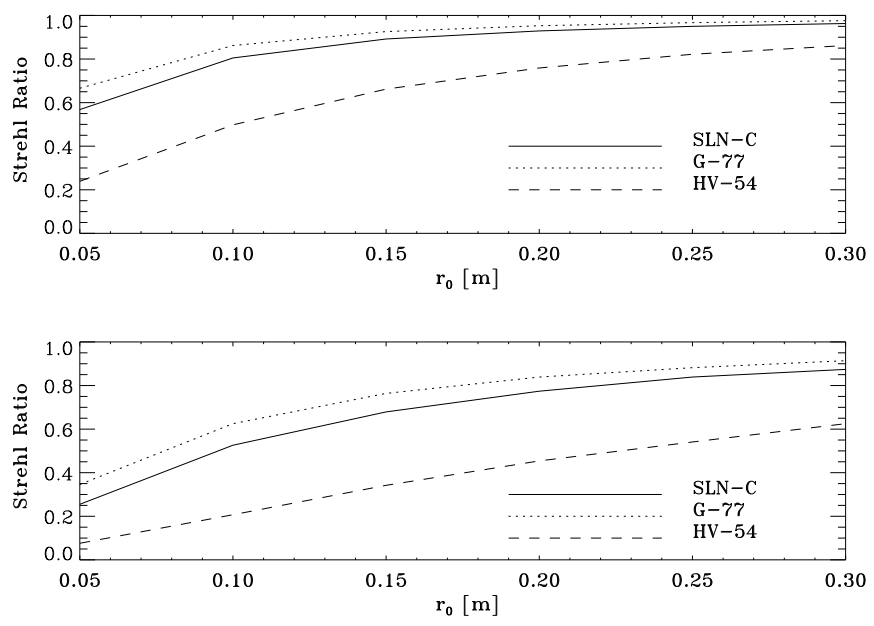

Fig. 4. Behaviour of $S R$ vs. $r_{0}$ for $D=3.58 \mathrm{~m}$ (upper panel) and $D=8.00 \mathrm{~m}$ (lower panel) telescope

\section{Conclusions}

Powerful mesospheric LGSs could give in the near future a strong impulse to the astronomical imaging at diffractionlimited resolution allowing full-sky coverage. With this goal in mind and taking into account the amount of efforts to erect and operate large telescopes one should point out in detail the fundamental limitation of any absolute tilt recovery technique especially under normal seeing conditions.

The results of our simulation show how in the visible range and for the $D=8.00 \mathrm{~m}$ telescope case, even under exceptional good seeing, the effects of the finite height of the mesospheric sodium laser in the multi-colour LGS tilt recovery approach are not completely negligible. Under normal seeing conditions the effect is even more severe, being the Strehl degradation of the order of $0.1 \ldots 0.6$.

The situation changes substantially for the $D=3.58 \mathrm{~m}$ case where Strehl degradation lower than 0.5 appears to occours rarely.

It should be pointed out that the details of the results depend upon the adopted atmospheric model choosen. An atmospherical situation where the spreading of the heights of the disturbing layers is kept to a minimum is less affected than situations where significant layers are placed at strongly different altitudes. This last statement translates into the fact that, at least using multicolour LGSs as tilt-recovery technique, care is to be given to the minimization of the ground layer seeing and that high altitude observing sites can have better results.

The effects of the variation of the effective layer height on the other proposed techniques for the absolute tilt determination should be carefully investigated, together with other types of fundamental limitations, making a clear distinction between the effects due to technologically-limited conditions (e.g. the number of photons per subaperture impose a fundamental limit due to the photon shot noise, but it is related to the power of the laser used to generate the LGS) and the ones strictly inherent to the concept described by the proposed technique.

Finally it is to be pointed out that one could make some additional measurements on the multicolour LGS in order to solve or to minimize the described effects, for example combining this technique with others.

Acknowledgements. Thanks are due to S. Esposito, of the Arcetri Astrophysical Observatory (Italy), for useful hints on the original manuscript.

\section{References}

Beckers J.M., 1993, ARA\&A 31, 13

Belen'kii, 1995, SPIE Proc. 2471, 289

Drummond J.D., Christou J.C., Fugate R.Q., 1995, ApJ 450, 380

Foy R., Boucher Y., Fleury B., et al., 1992, ESO Conf. Proc. 42,437 
Foy R., Labeyrie A., 1985, A\&A 152, L29

Foy R., Migus A., Biraben F., et al., 1995, A\&AS 111, 569

Fried D.L., Belsher J.F., 1994, JOSA-A 11, 277

Greenwwood D.P., 1977, JOSA 67, 390

Happer W., MacDonald G.J., Max C.E., Dyson F.J., 1994, JOSA-A 11, 263

Hufnagel R.E., 1974, OSA Technical Digest Series, paper WA1

Marchetti E., Ragazzoni R., 1996 (to appear on A\&AS)

McCullough P.R., Fugate R.Q., Christou J.C., et al., 1995, ApJ 438, 394

Miller M., Zieske P., Hanson D., 1976, SPIE Proc. 75, 30

Mountain M., Kurz R., Oschmann J., 1994, SPIE Proc. 2199, 41

Olivier S.S., Max C.E., Gavel D.T., Brase J.M., 1993, ApJ, 407, 428
Olivier S.S., An J., Avicola K., et al., 1995, in Adaptive Opt. 23, OSA Technical Digest Ser., p. 35

Pilkington J.D.H., 1987, Nat 330, 116

Ragazzoni R., 1996a, A\&A 305, L13

Ragazzoni R., 1996b, ApJ 465, L73

Ragazzoni R., Esposito S., Marchetti E., 1995, MNRAS 276, L76

Rigaut F., Gendron E., 1992, A\&A 261, 677

Roggermann M.C., Welsh B.M., Montera D., Rhoadarmer T.A., 1995, Appl. Opt. 34, 4037

Sandler D.G., Stahl S., Angel J.R.P., Lloyd-Hart M., McCarthy D., 1994, JOSA A 11, 925

Sarazin M., Roddier F., 1990, A\&A 227, 294

Tallon M., Foy R., 1990, A\&A 235, 549 